\title{
Bitter colloid observations of magnetic structures in perpendicular magnetic recording media
}

\author{
St. Porthun, P. ten Berge, J.C. Lodder \\ MESA Research Institute, University of Twente, P.O. Box 217, NL-7500 AE Enschede, The Netherlands
}

Received 23 September 1992; in revised form 23 November 1992

In this study the Bitter colloid method has been used for domain structure observation in high coercivity $\mathrm{Co}-\mathrm{Cr}$ and for observations of magnetization state of single alumite needles. Furthermore the results of bit writing experiments in $\mathrm{Co}-\mathrm{Cr}$ have been observed by this method, which allows to compare samples, prepared with different parameters.

\section{Introduction}

Investigations on magnetic storage media require methods for observation of magnetic structures. In this study, where high coercivity $\mathrm{Co}-\mathrm{Cr}$ films with perpendicular anisotropy and alumite, a model material for perpendicular recording are investigated, a high resolution method is needed in order to resolve magnetic structures smaller than $100 \mathrm{~nm}$.

The Bitter colloid method allows to observe the influence of external fields on the domain structure of a magnetic material. It uses ferrofluids for imaging of the stray fields of the sample magnetization. Ferrofluids consist of water or an inorganic liquid carrier containing magnetic particles, usually $\mathrm{Fe}_{3} \mathrm{O}_{4}, \gamma-\mathrm{Fe}_{2} \mathrm{O}_{3}$ or metallic particles of iron, nickel or cobalt having 10-15 nm diameter, which are responsible for the magnetic behaviour of the fluid. The particles interact by magnetic forces and by electrostatic and van der Waals forces. Liquid dispersants are added which surround the particles and lead to a steric repulsion between them, which avoids a particle clustering in the ferrofluid.

Compared to other technical applications for

Correspondence to: St. Porthun, MESA Research Institute, University of Twente, P.O. Box 217, NL-7500 AE Enschede, The Netherlands. magnetic structure imaging the used ferrofluids are higher diluted and have weaker steric repulsion between the particles. Dilutions of water based ferrofluids (which gave the best results in Bitter technique) form particle clusters after storing them for several days.

For the Bitter colloid method the fact is used, that the equilibrium of repulsive steric forces and attractive van der Waals and magnetic forces is influenced by the stray field of the sample magnetization, leading to differences in colloid particle densities in the ferrofluid. Although these concentration changes in the fluid can already be used for dynamic observations in an optical microscope, for technical reasons (in order to be comparable to SEM images) the carrier liquid has always been removed by drying or rinsing, so that the colloid particles remain on the surface, forming Bitter patterns.

Theoretical descriptions of the Bitter pattern formation are given by Kittel [1,2] and Hartmann [3]. As shown by Craik [4] in absence of external ficlds the colloid particles collect above domain transitions (see fig. 1a), and in a sufficiently high external field the domains in field direction are completely covered (fig. 1b).

The resolution of the method depends on ferrofluid parameters as well as on surface parameters of the investigated layers. The most important properties of ferrofluids for high resolution 
observation are the strength of their steric system and their surface wettability. Ferrofluids with a weak steric system can form reversible agglomerates, which form multidomain particles and have a higher field sensitivity as described by Yeh in [5]. Especially in weak stray fields an insufficient surface wettability can influence the formation of Bitter patterns during colloid drying [10]. Rough surfaces are a kinctic barrier for the mobility of the particles and cause stray fields which affect the stray ficlds of the sample magnetization.

In this study the Bitter method has been used for visualisation of small magnetic structures in high cocreivity $\mathrm{Co}-\mathrm{Cr}$ layers and alumite as well as for observations of bit writing properties of the layers.

\section{Experimental}

The ferrofluids used in this study are EMG 508 [6], and Magnetol [7]. Both ferrofluids contain iron oxide particles of $10-15 \mathrm{~nm}$ diameter. EMG 508 is based on water; Magnetol is a dilution of a fluid for engineering applications. It is based on Kerosene, the carrier has to be rinsed in Nonane $\left(\mathrm{C}_{9} \mathrm{H}_{20}\right)$ to get a dried Bitter pattern.

Before covering the sample surface with ferrofluid the samples have been saturated and the field has been reduced to the observation field valuc. For obtaining a high resolution observation it is essential that the external applied field does not change any more when the ferrofluid is on the sample. The $1 \times 1 \mathrm{~cm}^{2}$ sample is covered by ferrofluid completely and then the sampleholder and magnet are tilted $60^{\circ}$ for colloid drying. This gives a good homogeneity of the ferrofluid layer and a slope in layer thickness. The optimum colloid concentration has to be found empirically. It depends on the sample and differs from 0.1 vol\% magnetic material for SEM observations on alumite to $2.5 \mathrm{vol} \%$ for optical microscope observations of bit tracks on Co-Cr. Generally a higher concentration increases the contrast, but decreases the resolution of the method.

For the observation of written tracks and for overview pictures we used a Leitz MM5 light microscope. The used magnification on photo sheets was 400 times, using Polaroid 52 PolaPan $4 \times 5$ instant sheet film and a $32 \times / 0.60$ bright field objective.

Finer details on the Bitter patterns were observed by a Scanning Electron Microscope. This Bitter colloid-SEM method was first used by Goto [8]. We used a dual stage SEM (ISI ABT DS-130C) detecting secondary electrons. In order to get surface information relatively low acceleration voltages of $7-3 \mathrm{kV}$ have been applied. To avoid surface charging a layer of $\mathrm{Au}-\mathrm{Pd}$ was sputtered on the Fe-alumite samples.

The experimental set-up, used for bit writing in $\mathrm{Co}-\mathrm{Cr}$ is an $\mathrm{X} / \mathrm{Y}$ table with electric stepper motors with $100 \mathrm{~nm}$ precision. The tracks were written by hard disk heads in contact recording with maximum head to medium specd of 48 $\mu \mathrm{m} / \mathrm{s}$.

\subsection{Domains in $\mathrm{Co}-\mathrm{Cr}$}

We have used the Bitter colloid-SEM method to observe the intrinsic domain structure of high cocreivity $\mathrm{Co}-\mathrm{Cr}$ samples. The investigated layers were prepared by RF-sputtering on a Ge underlayer on a Si-wafer. They consist of perpendicularly oricnted crystallites, having a magnetic anisotropy perpendicular to the film plane. More information about the $\mathrm{Co}-\mathrm{Cr}$ layers is given in [9].

First of all a Bitter pattern on a low coercivity sample is given in fig. 2. The bright areas are

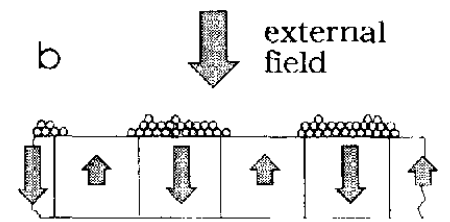

Fig. 1. Colloid covering of domain transitions without external field (a) and Colloid covering of domains, which are directed as the external field (b). 


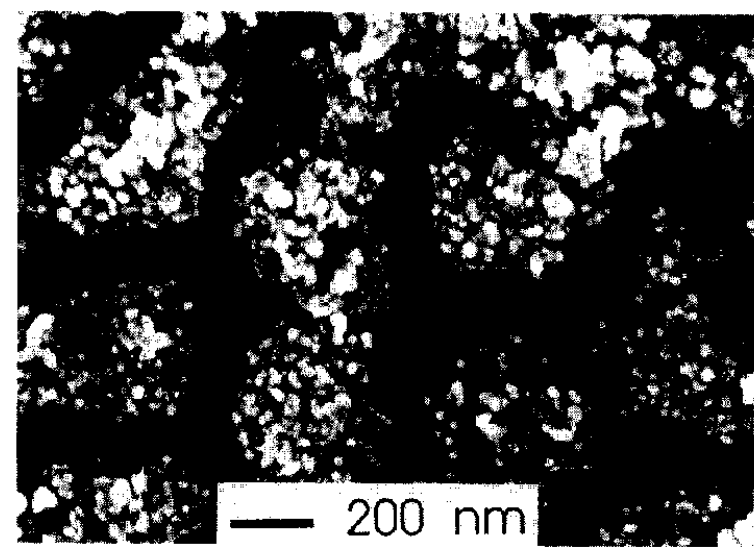

Fig. 2. Bitter pattern on a low coercivity $\mathrm{Co}-\mathrm{Cr}$ sample $H_{\text {ext }}=+80 \mathrm{kA} / \mathrm{m}$ (anhysteretic state, reached by superposing a decreasing ac field to $H_{\text {ext }}$ ) obscrved by SEM.

covered by colloid particles (particle diameter $\approx 15 \mathrm{~nm}$ ) and show the domains magnetized in direction of the external magnetic field. The dark regions (uncovered) are domains magnetized op-
Table 1

Data of samples used for intrinsic domain imaging

\begin{tabular}{llccl}
\hline Sample & $\begin{array}{l}M_{\mathrm{s}} \\
(\mathrm{kA} / \mathrm{m})\end{array}$ & $\begin{array}{l}\mathrm{H}_{\mathrm{c} \perp} \\
(\mathrm{kA} / \mathrm{m})\end{array}$ & $H_{\mathrm{c}} / H_{\mathrm{k}}$ & $\begin{array}{l}\text { Co-Cr layer } \\
\text { thickness [nm] }\end{array}$ \\
\hline low $H_{\mathrm{c} \perp}$ & 402 & 8 & 0.015 & 520 \\
high $H_{c,}$ & 500 & 107 & 0.252 & 100 \\
\hline
\end{tabular}

posite to the external field. Here the $\mathrm{Co}-\mathrm{Cr}$ crystallites (columns) are visible. The irregularity at the borders of the domains is caused by the shape of the agglomerates and extends over the opposite (dark) domains.

For observation of the smaller structures on high coercivity samples this can become a limiting factor as visible in fig. 3a (same scale as fig. 2). Here the structure period is about $200 \mathrm{~nm}$ and is much worse resolved. The magnetic properties of both samples are given in table 1 .

In order to test to what degree these patterns are affected by nonmagnetic processes, two more
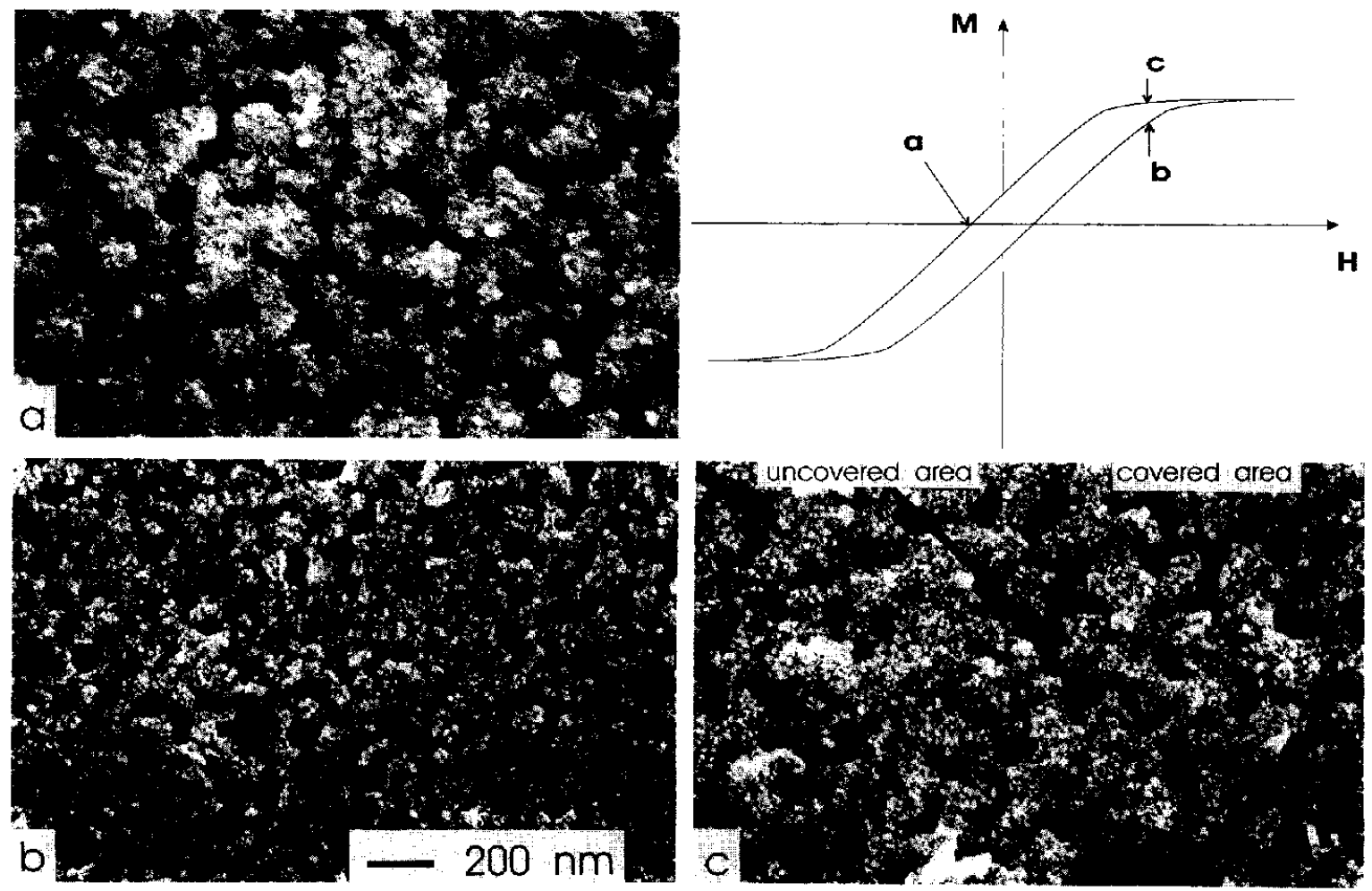

Fig. 3. SEM observation of Bitter colloid pattern on high coercivity $\mathrm{Ca}-\mathrm{Cr}$ sample in: (a) $H_{\mathrm{appl}}=-107 \mathrm{kA} / \mathrm{m}=-I H_{\mathrm{c},\lrcorner}$, (b) $H_{\text {appl }}=-288 \mathrm{kA} / \mathrm{m}$ and $(\mathrm{c}) H_{\mathrm{appl}}=-288 \mathrm{kA} / \mathrm{m}$. 
Table 2

Dala of $\mathrm{Co}-\mathrm{Cr}$ samples

\begin{tabular}{|c|c|c|c|c|c|c|c|c|}
\hline Sample \# & $\begin{array}{l}\text { Thickness } \\
\text { (nm) }\end{array}$ & atr/e $\mathrm{Cr}$ & $\begin{array}{l}M_{s} \\
(\mathrm{k} \Lambda / \mathrm{m})\end{array}$ & $\begin{array}{l}H_{6-} \\
(\mathrm{kA} / \mathrm{m})\end{array}$ & $\begin{array}{l}H_{\mathrm{elli}} \\
(\mathrm{k} \Lambda / \mathrm{m})\end{array}$ & $\begin{array}{l}H_{\mathrm{k}} \\
(\mathrm{k} \Lambda / \mathrm{m})\end{array}$ & $\left(M_{\mathrm{T}} / M_{\mathrm{s}}\right)_{\|}$ & $Q$ \\
\hline 911028 & 100 & 23 & 318 & 64.0 & 9.0 & & 0.05 & \\
\hline $910716 \mathrm{a}$ & 100 & 19 & 508 & 47.2 & 11.8 & 420.4 & 0.09 & 0.83 \\
\hline 910514 & 100 & 19 & 478 & 97.7 & 55.3 & 164.8 & 0.54 & 0.27 \\
\hline 911122 & 80 & 23 & 417 & 53.9 & 8.3 & & 0.05 & \\
\hline
\end{tabular}

Bitter pattern observations have been made in two different magnetization states for the same external field (fig. $3 b$ and $3 c$ ). Besides the sample magnetization state all other parameters are kept constant this way. In fig. $3 b$ there are many dark (no colloid) bubble shaped domains, the sample has not been saturated yet. The maximum field value that could be applied to the sample was about $660 \mathrm{kA} / \mathrm{m}$, which did not completely saturate it $\left(H_{\mathrm{k}}=424 \mathrm{kA} / \mathrm{m}\right)$. This explains, why fig. $3 c$ still shows a few bubble domains. On these photographs a precise measurement of a domain period as it is done by Šimšová et al. on low coercivity $\mathrm{Co}-\mathrm{Cr}$ samples [10-14] is difficult be- cause the domain boundary is not defined exactly by the colloid for such small structures.

Considering that all external conditions are the same in both observations one can conclude that with this ferrofluid it is possible to image these small magnetic structures.

\subsection{Bit writing in $\mathrm{Co}-\mathrm{Cr}$}

In order to investigate their writing properties bits have been written on $\mathrm{Co}-\mathrm{Cr}$ samples and observed by Bitter colloid technique. The properties of the $\mathrm{Co}-\mathrm{Cr}$ samples used for these writing experiments are given in table 2 . The data are
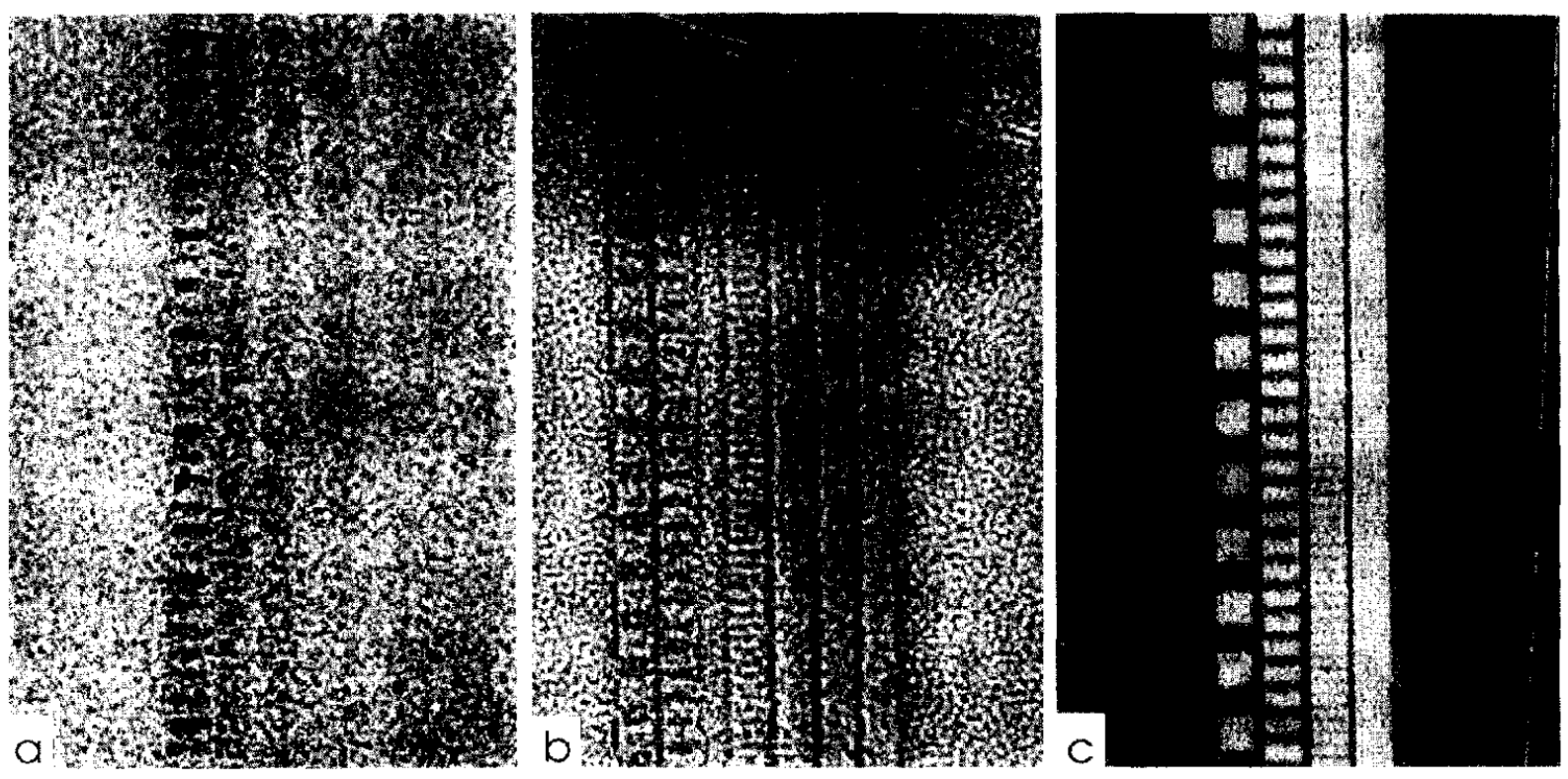

$25 \mu \mathrm{m}$

Fig. 4. Light microscope photograph of bit tracks. (a) On sample 910514 (layer thickness $100 \mathrm{~nm}$ ); writing densities from left to right $0.1,0.25,0.5$, and $1 \mathrm{kbpmm}$. (b) On sample $910716 \mathrm{a}(100 \mathrm{~nm})$; writing densities from left to right $0.125,0.25,0.5,1,1.33$ and 2 kbpmm. (c) On sample $911028(100 \mathrm{~nm})$; writing densitics from left to right $0.1,0.25,0.5$, and $1 \mathrm{kbpmm}$. 

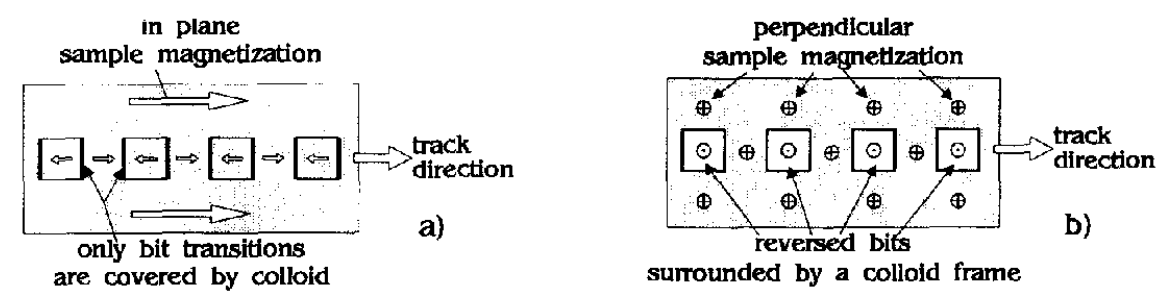

Fig. 5. Colloid deposition on bit tracks in materials with in-plane (a) and perpendicular (b) anisotropy.

derived from vibrating sample magnetometer (VSM) and torque meter measurements.

On each sample tracks with $0.1,0.25,0.5$ and 1 kbpmm have been written using optimum writing currents and visualised by Bitter colloid and light microscope. The bit patterns have been compared for sample 910514 (fig. 4a), for $910716 a$ (Fig. 4b, both 19 at\% Cr)and for 911028 (fig. 4c, 23 at.\% $\mathrm{Cr}$ ). Because of their $\mathrm{Cr}$ amount of 19 at $\%$ the relation $Q$ of anisotropy and demagnetizing energy $\left(Q=K_{1} / \frac{1}{2} \mu_{0} M_{\mathrm{s}^{2}}\right)$ is smaller than 1 , thus an overall in-plane anisotropy should be favourable. According to Wielinga [15] $\mathrm{Co}-\mathrm{Cr}$ samples show perpendicular anisotropy already above $Q \approx 0.8$, because the sample is not in uniform magnetization state as considered in the demagnetizing energy term. Sample 910514 has a high dispersion $\left(\approx 14^{\circ}\right)$ of the $c$-axis and irregularly shaped $\mathrm{Co}-\mathrm{Cr}$ columns. The high $\left(M_{\mathrm{r}} / M_{\mathrm{s}}\right)_{\|}$ value and the low $Q$ suggest in-plane anisotropy, which is pointed out by the colloid stripes on the upper track of fig. 4a (also see fig. 5a). Sample 910716a with $Q=0.82$ already shows perpendicular anisotropy in fig. $4 \mathrm{~b}$. The switched bits are surrounded by a frame of colloid. Figure $4 \mathrm{c}$ for 911028 shows really perpendicular writing behaviour. The bright areas are switched in their magnetization direction opposite to the magnetization of the previously saturated sample. The bits are surrounded by a colloid frame (see fig. $5 b)$.

\subsection{Depth distribution of magnetization in written bits}

During this work an attempt of investigation of depth profile of written bits has been made. In order to visualize the magnetization deeper in the material by Bitter colloid method steps have been etched into the sample by ion beam etching (IBE) after writing the bits. This method was used by Iwasaki in [16] for testing writing head performance. Its principle is shown schematically in fig. 6 .

Care had to be taken for some experimental parameters to reach results which allow conclusions about the sample magnetization: Etching in short intervals with long breaks in between makes sure that the sample magnetization is not changed thermally. Since the etching rates for $\mathrm{Co}$ and for $\mathrm{Cr}$ do not differ significantly their ratio is not expected to be changed. However, due to the ion bombardment local changes in chemical composition can occur. AFM measurements show a comparable roughness on etched and unetched surfaces, so the Bitter colloid will give comparable results.

A step of $40 \mathrm{~nm}$ depth has been etched in Co-Cr sample 911122. The Bitter colloid observation is given in fig. 7 and compared to sample 910716a (fig. 8, data of both samples is given in table 2).

From this experiment conclusions about the depth profile of magnetic tracks may be drawn.

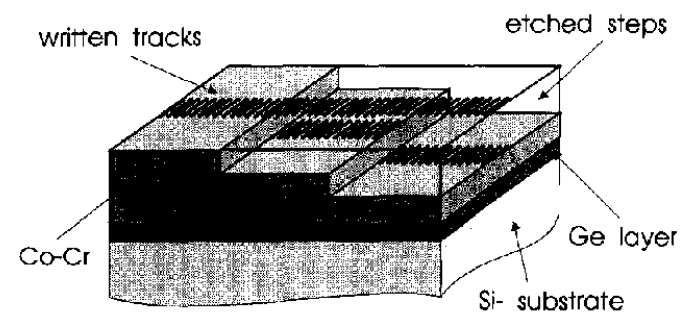

Fig. 6. Ion beam etching of steps for magnetization imaging inside the layer by Bitter colloid. The transparent regions are etched away by IBE. 

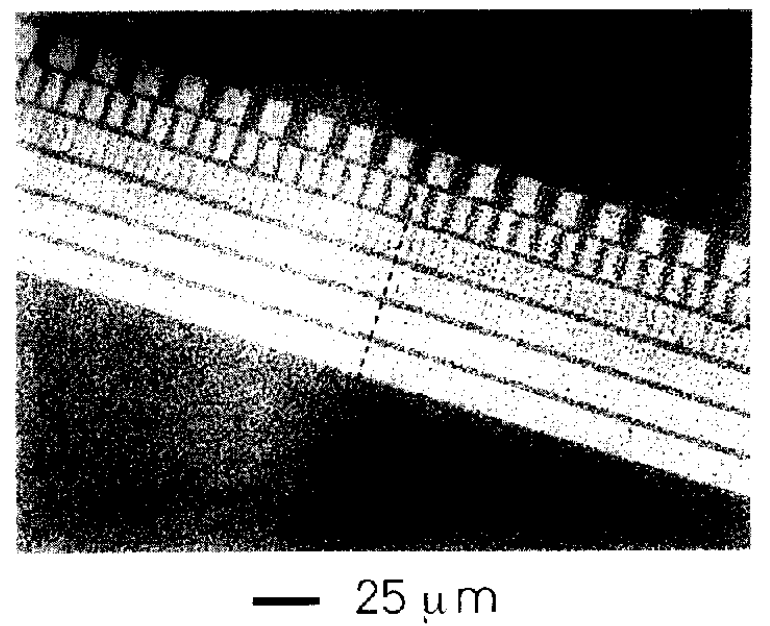

Fig. 7. Light microscope photograph of bit tracks $0.125,0.25$, $0.5,1,1.33$ and $2 \mathrm{kbpmm}$ (from top downwards) on 911122 ( 80$)$ nm layer thickness) unetched (dark right area) and with a 40 nm step (left bright area).

The samples having an overall in plane magnetic anisotropy can be written only in the uppermost regions of the layers. The perpendicular anisotropy sample is magnetized through the whole layer during magnetic writing.

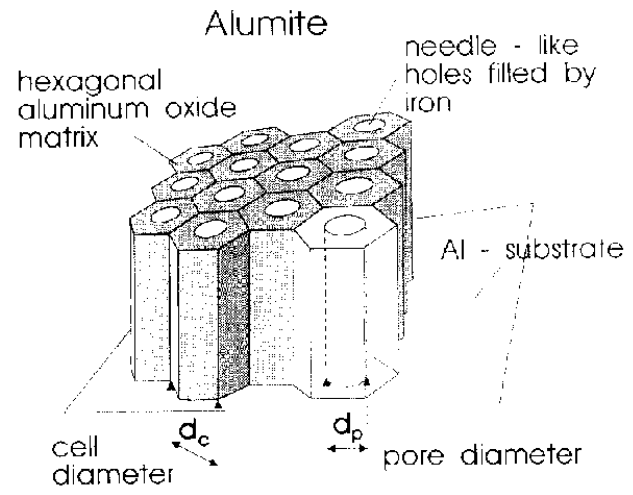

Fig. 9. Structure of alumite.

\subsection{Magnetic structures in Fe-alumite}

A very useful material for challenging the Bitter colloid/ SEM method is alumite. The structure of alumite is shown in fig. 9. It consists of a hexagonal aluminium oxide cell structure containing ferromagnetic needles. The challenge for the Bitter colloid method lies in the small structure size of the alumite needles which can be varied in a region from $250 \mathrm{~nm}$ down to $20 \mathrm{~nm}$ cell diameter $\left(d_{\mathrm{c}}\right)$. This region becomes difficult to resolve
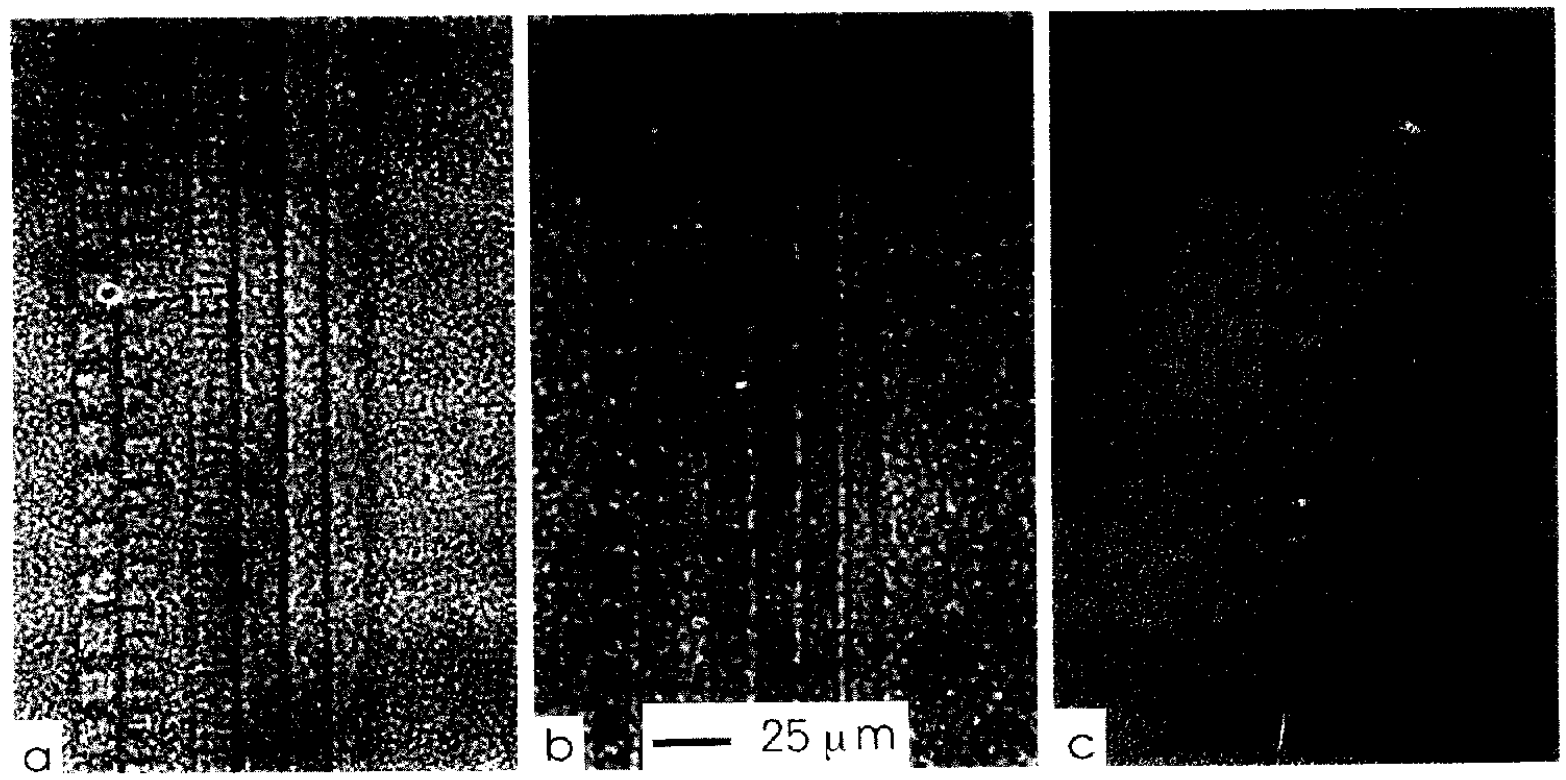

Fig. 8. Bitter colloid pattern on etched surfaces in different depths in sample 910716 (unetched surface given in fig. $4 \mathrm{~b}$ ); (a) $20 \mathrm{~nm}$ step (thickness $80 \mathrm{~nm}$ ), (b) $40 \mathrm{~nm}$ step ( $60 \mathrm{~nm}$ thick) and (c) $60 \mathrm{~nm}$ step ( $40 \mathrm{~nm}$ thick). 
by the Bitter colloid method. However, alumite gives good contrast between the iron needles and the aluminium oxide matrix in the SEM and has a very hard surface, which can be used many times for colloid preparations without damaging.

A lot of work has been done about the dependence of the magnetic behaviour of alumite on the morphology parameters $[17,18]$. The magnetic reversal mode in alumite is found to be curling, but the angular dependence of the coercivity suggests particle interactions [19]. Calculations of demagnetizing effects [20] and magnetic measurements [21] have been carried out for investigation of magnetic reversal in alumite.

Alumite is used as a model material for perpendicular recording which we use to experimentally test theories about magnetic switching behaviour of systems of shape anisotropic particles. For this, it is important to investigate the magnetic structure of alumite in external magnetic fields and the magnetization of single alumite needles.

The data of the alumite sample investigated here are given in table 3 . Relatively large ncedle size and good substrate conductivity made it very useful for SEM observation method.

\subsubsection{Magnetic reversal observation on alumite}

In order to test the suitability of the Bitter colloid method for alumite needle observations Bitter patterns have been prepared in different external fields and compared to the VSM hysteresis loop of the sample. Before each observation the sample has been saturated in one field dircction (here called positive field direction). Then the external field has been reduced to the observation field value. In the resulting Bitter patterns (as given in fig. 12) some alumite needles are completely covered by colloid and some are uncovered. According to fig. $1 \mathrm{~b}$ in a sufficiently strong external field always the needles which are

Table 3

Alumite sample data

\begin{tabular}{llllll}
\hline & $\begin{array}{l}d_{\mathrm{c}} \\
(\mathrm{nm})\end{array}$ & $\begin{array}{l}d_{\mathrm{p}} \\
(\mathrm{nm})\end{array}$ & $p$ & $\begin{array}{l}M_{\mathrm{s}} \\
(\mathrm{kA} / \mathrm{m})\end{array}$ & $\begin{array}{l}H_{\mathrm{c} \perp} \\
(\mathrm{kA} / \mathrm{m})\end{array}$ \\
\hline $\mathrm{W} 2 \# 2$ & 246 & 125 & 0.23 & 176 & 27.9 \\
\hline
\end{tabular}

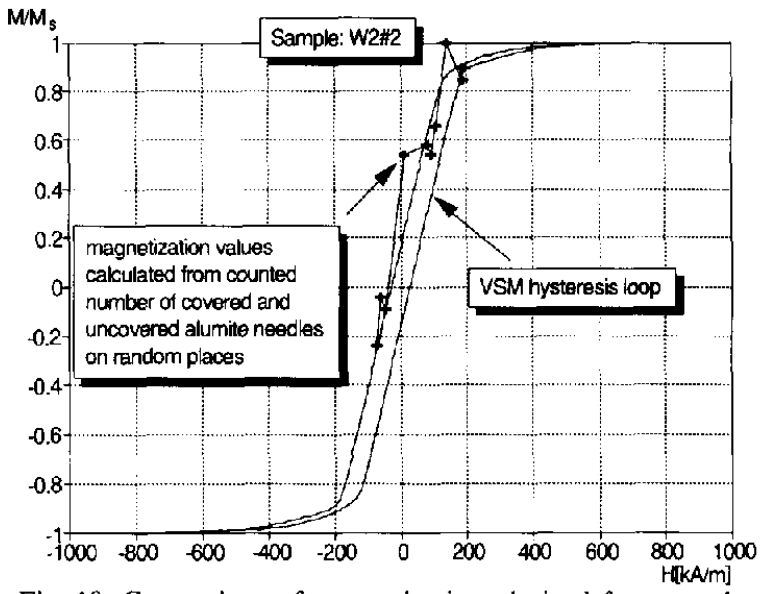

Fig. 10. Comparison of magnetization, derived from number of switched and unswitched needles to VSM hysteresis loop.

magnetized in field direction should be covered. This means, the reversed needles are uncovered as long as the external field is positive and covered in negative external fields. In a series of colloid patterns, prepared in different external fields, on randomly chosen places on the sample the relation between the number of reversed needles and the total number of needles on the photograph has been related to the magnetization state of the whole sample by calculating an $\left(M / M_{\mathrm{S}}\right)^{*}$ by the relation

$$
\left(M / M_{\mathrm{S}}\right)^{*}=2 \frac{\# \text { reversed needles }}{\# \text { all needles }}-1 \text {. }
$$

The values of $\left(M / M_{\mathrm{S}}\right)^{*}$ have been compared to the corresponding hysteresis loop measured by VSM. This is shown in fig. 10.

The deviations between the $\left(M / M_{\mathrm{S}}\right)^{*}$ curve and the hysteresis loop can be explained by the simplicity of the switching model which is considered here for the calculation of $\left(M / M_{\mathrm{S}}\right)^{*}$ : All needles are assumed to have the same size and magnetic moment. Their magnetization can only orient parallel or antiparallel to the film normal.

Another reason for this deviation is the limited number of counted needles (about 100-150), which is very small compared to the overall amount of needles, measured by VSM. Besides that there are several holes in the aluminium oxide matrix not filled completely by iron. They 


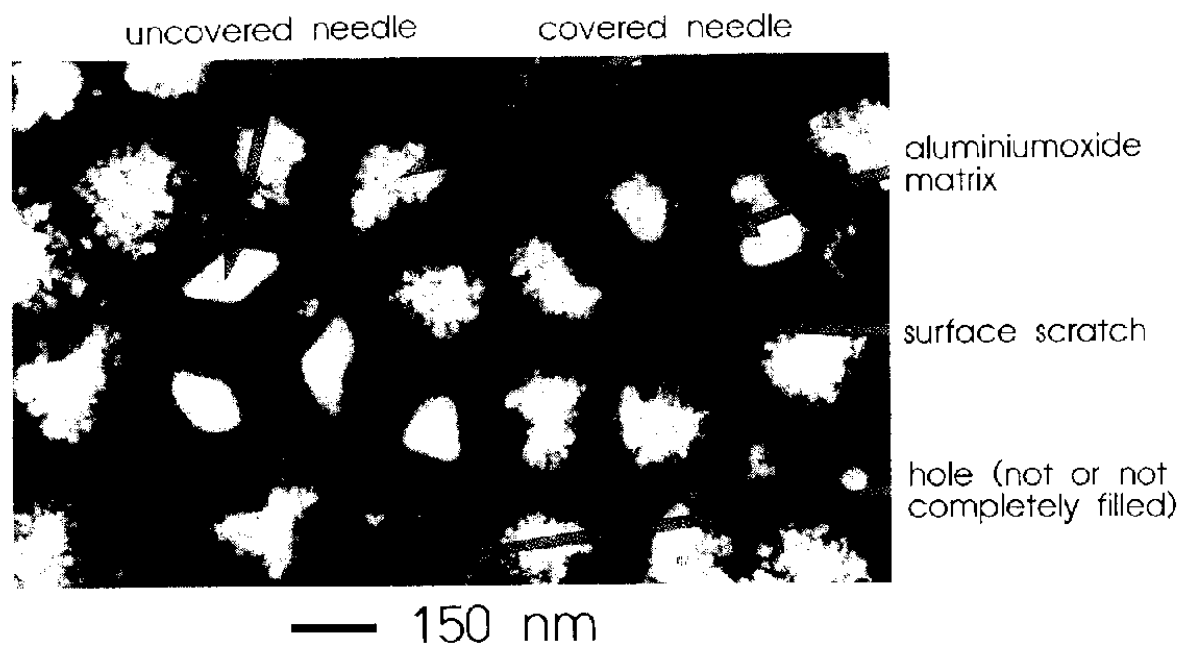

Fig. 11. SEM photo of the investigated area on sample $\mathrm{W} 2 \# 2$ in $+80 \mathrm{kA} / \mathrm{m}$ external field.

are not visible in the SEM pictures and not sensed by the Bitter colloid but give a contribution to the VSM hysteresis loop. Nevertheless an overall agreement between the $\left(M / M_{\mathrm{S}}\right)^{*}$ curve and the hysteresis loop is clear, which means that Bitter colloid SEM method can resolve the magnetization state of alumite needles having $125 \mathrm{~nm}$ diameter.

\subsubsection{Individual needle behaviour}

For this experiment the point of interest was to have a closer look at the individual needles, at their switching fields and at the interactions between them. In order to be able to compare magnetic configurations, caused by different dry- ing fields and to reproduce patterns some series of photos were taken at the same area at the sample. Because it is possiblc to relocate individual needles this allows to observe the magnetic state of individual needles in different external fields by Bitter colloid. The area on the sample where the observations are made on is shown in fig. 11. The following experiments were made to characterize the magnetization state of 39 alumite needles on this figure.

For a better overview this region is drawn in the following figures and the needles are numbered. Reversed needles are filled black. Needles, which are half covered by colloid or where it is not clearly visible whether they are reversed,
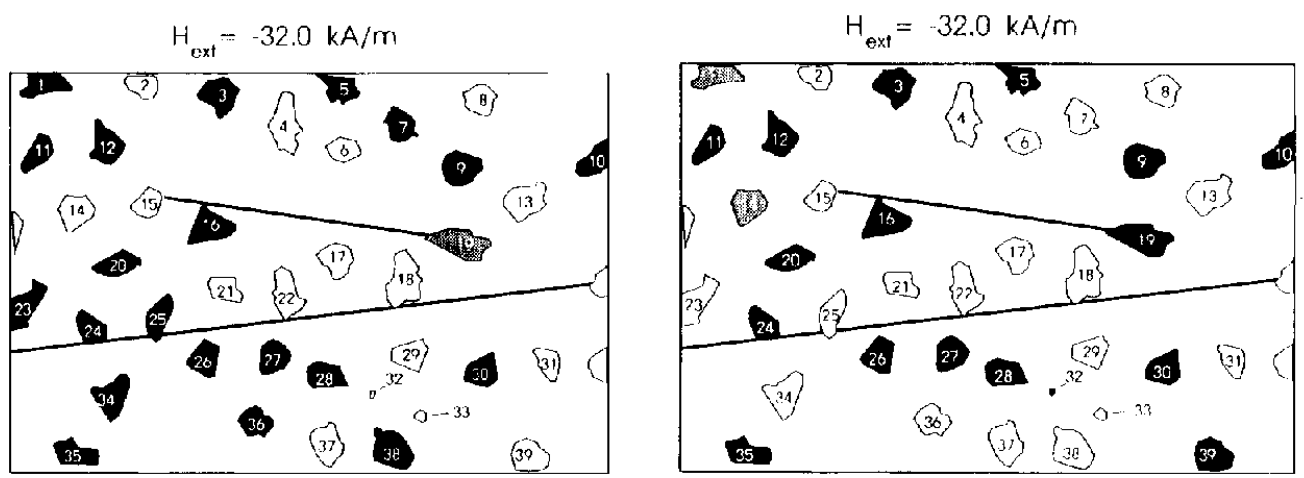

Fig. 12. Reproducibility test of Bitter patterns in the same external field. The sample has been saturated in between. 
are filled grey. White needles are still magnetized in the direction parallel to the previous saturation.

In order to test the reproducibility of the structures the Bitter colloid preparation has been made twice in the same external field, as shown in fig. 12. Six needles (No. 7, 25, 34, 36, 38 and $39)$ are found in a different magnetization state. For three needles no conclusions about the magnetization state could be made in one of the corresponding Bitter patterns, so that they are drawn grey here (No. 1, 14 and 19). For the remaining 30 needles the magnetization state has been reproduced.
In fig. 13 a series of preparations in different external fields is shown. The aim of this experiment was to observe the magnetization state of the needles in different fields and to draw conclusions about the switching field of each needle. The series starts at positive saturation, where all needles are covered and have an aligned magnetization. With decreasing field more and more ncedles switch back.

Although these pictures are taken in different magnetization cycles (the sample has been saturated at least once in-between) they show a certain sequence of necdle switching. Several groups of needles tend to reverse in the same field,
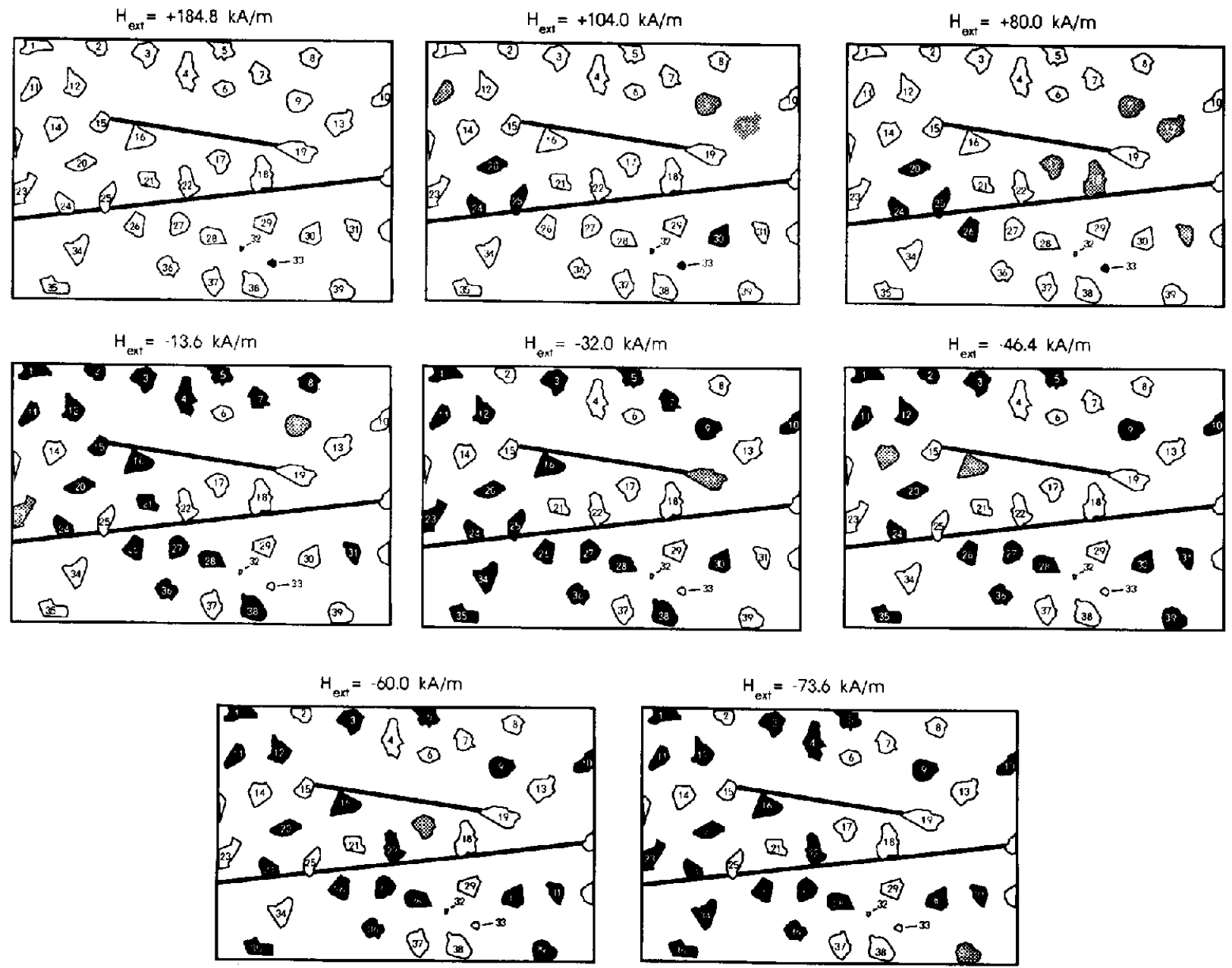

Fig 13. Magnetization state of alumite needles on sample W2\#2. 
which could be an indication for a domain like reversal. Examples for such groups are needles $(20,24,26)$ and $(26,27,28,36)$.

In contradiction to this in an ideal hexagonal alumite structure one should expect the needles to reverse randomly, because the flux closure of a switched needle strengthens the position of the surrounding unswitched needles. In opposition to that the micromagnetic simulations of van Kooten [22] on arrays of perpendicular columnar $\mathrm{Co}-\mathrm{Cr}$ structures suggest collective reversals. The neighbour ncedles interact by the in plane field component of the top layer of the needles, where the magnetization is inclined during the reversal. This reversal mechanism is also thinkable for the needles of alumite.

Furthermore the used alumite sample has very many structure faults such as holes in the matrix which are not completely or not at all filled by an iron needle, differences in needle size $\left(d_{\mathrm{c}}\right)$ and in next neighbour distances $\left(d_{\mathrm{p}}\right)$. These irregularities will cause differences in the energy barrier for reversal, so that the results can not be used to prove models or calculations where a regularity of the structures is assumed.

\section{Conclusions}

The resolution of the Bitter colloid SEM method we used is better than $100 \mathrm{~nm}$. It is limited by concentration inhomogeneities, which arise during the ferrofluid drying. Due to the more advantageous agglomeration properties we reached best results with water based ferrofluids. Domains on high coercivity $\mathrm{Co}-\mathrm{Cr}$ samples and magnetization states of single needles of an alumite sample having a needle diameter of $125 \mathrm{~nm}$ have been observed.

Because it only needs standard equipment the method is relatively cheap, but it provides a higher resolution than digitally enhanced Kerr technique. A disadvantage of the method is that it is a non-dynamical technique. The magnetic origin of the powder patterns has to be checked through observation of series of single experiments.

\section{Acknowledgement}

The authors thank the CAMST (Community Action on Magnetic Storage Technology) EC stimulation project for their financial support.

\section{References}

[1] C. Kittel, Phys. Rev. 76 (1949) 1527.

[2] C. Kittel, Phys. Rev. 21 (1949) 541-583.

[3] U. Hartmann and H.H. Mende, Z. Phys. B - Condensed Matter 61 (1985) 29-32.

[4] D.J. Craik, Brit. J. Appl. Phys. 17 (1966) 873-877.

[5] N.H. Yeh, IEEE Trans, Mag. 16 (1980) 979-981.

[6] Ferrofluidics EMG 508, Ferrofluidics Corp., 40 Simon Street, Nashua, NH 03061 .

[7] N. Buske and H. Somntag, Mater. Sci. Forum 25-26 (1988) 111-124.

[8] K. Goto and T. Sakurai, Appl. Phys. Lett. 30 (1977) $355-356$

[9] P. ten Berge. J.C. Lodder, S. Porthun and Th.J.A. Popma, J. Magn. Magn. Mater. 11.3 (1992) 36-46.

[10] J. Šimšová, R. Gemperle, J.C. Lodder, J. Magn. Magn. Mater. 95 (1991) 85-94.

[11] J. Šimšová, J.C. Lodder, J. Kaczér, R. Gemperle, K. Jurek and I. Tomáš, J. Magn. Magn. Mater. 73 (1988) $131-1.35$.

[12] J. Šimšová, V. Kamberský, R. Gemperle, J.C. I ıdder. W.J.M.A. Geerts, B. Otten and P. ten Berge, J. Magn. Magn. Mater. 101 (1991) 196-198.

[13] J. Šimšová, R. Gemperle, J.C. Lodder, J. Kaczér, I. Murtinová, S. Saic and I. Tomáš, Thin Solid Films 188 (1990) 43-56.

[14] J. Šimšová, R. Gemperle, J. Kaczér, J.C. Lodder, IEEE Trans. Mag. 26 (1990) 30-32.

[15] T. Wielinga, Thesis, University of Twente, Enschede, The Netherlands (1983).

[16] S. Iwasaki and J. Hokkyo, Ohmsha Ltd., Tokyo (1991), Chapter 4

[17] Li Cheng-Zhang and J.C. Lodder, J. Magn. Magn. Mater. 88 (1990) 236-246.

[18] J.C. Lodder and Li Cheng-Zhang, IEEE Trans. Mag. 25 (1989) $4171-4173$.

[19] G.'T.A. Huysmans and J.C. Lodder, J. Appl. Phys. 64 (1988) 2016-2021.

[20] E.O. Samwel, P.R. Bissell and J.C. Lodder, J. Magn. Magn. Maler. 115 (1992) 327-332.

[21] E.O. Samwel, P.R. Bissell, J.C. Lodder. J. Appl. Physics, to appear.

[22] M. van Kooten, S. de Haan, J.C. Lodder A. I yberatos. R.W. Chantrell and J.J. Miles, J. Magn. Magn. Mater., to appear. 